
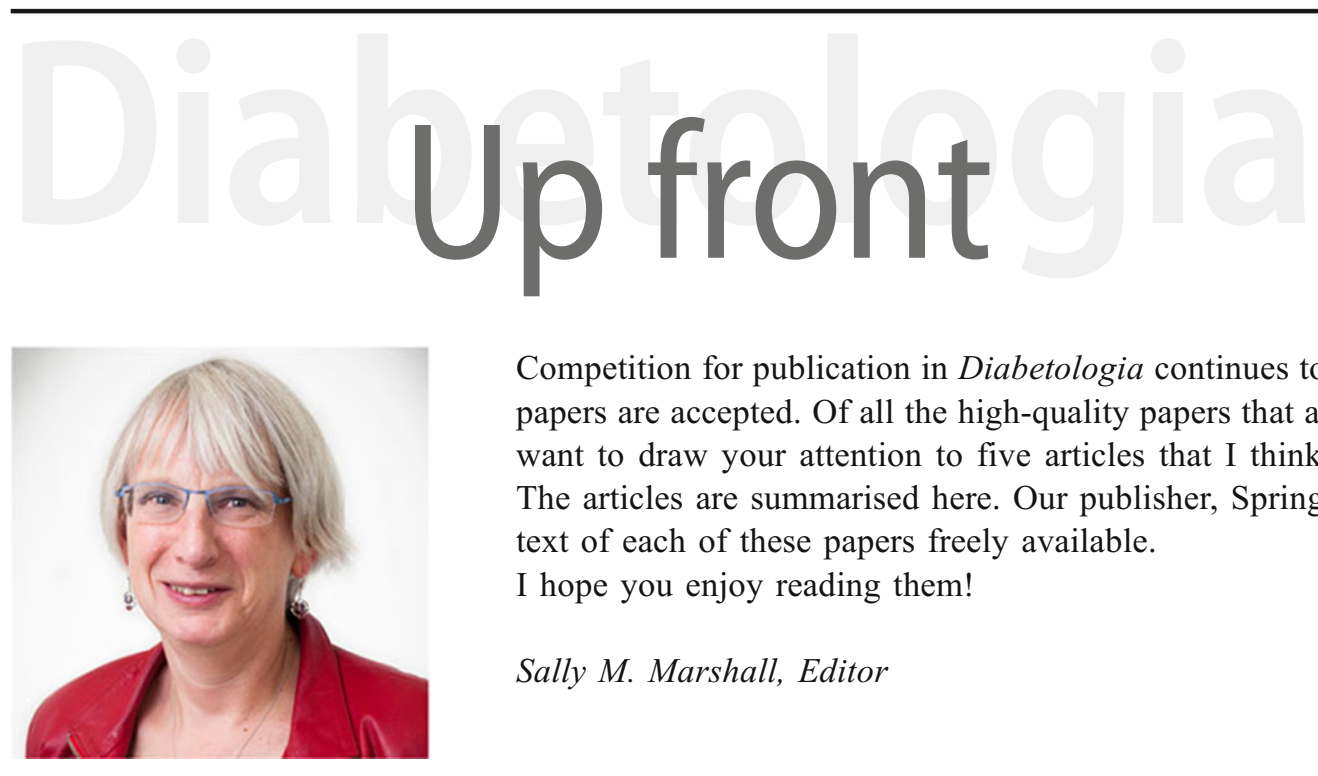

Competition for publication in Diabetologia continues to grow, and less than $20 \%$ of papers are accepted. Of all the high-quality papers that appear in this month's issue I want to draw your attention to five articles that I think are particularly interesting. The articles are summarised here. Our publisher, Springer, has kindly made the full text of each of these papers freely available.

I hope you enjoy reading them!

Sally M. Marshall, Editor

\section{A life course perspective on diabetes: developmental origins and beyond}

This issue features a special series of reviews on different aspects of diabetes throughout the life course. Jonathan Wells (https://doi.org/10.1007/s00125-019-4944-8) begins the series by considering diabetes risk from an evolutionary perspective to explain how adverse environments can increase diabetes risk and that this risk can be passed on to subsequent generations. Next, Golden et al (https://doi.org/10.1007/ s00125-019-4968-0) summarise racial/ethnic differences in the frequencies of type 2 diabetes across the life course, while Huebschmann et al (https://doi.org/10.1007/s00125-0194939-5) discuss the physiological and behavioural mechanisms that may underlie variations between the sexes with respect to diabetes prevalence and cardiovascular risk over a life time. The next two reviews by Stein et al (https:// doi.org/10.1007/s00125-019-4930-1) and Perng et al (https:// doi.org/10.1007/s00125-019-4914-1) focus on developmental under- and overnutrition, respectively, and obesity/diabetes risk. Fernandez-Twinn et al (https://doi.org/10.1007/s00125019-4951-9) look at how in utero exposures lead to the 'developmental programming' of offspring obesity and diabetes in later life via a variety of mechanisms, including epigenetics, while Sharp and Lawlor (https://doi.org/10.1007/ s00125-019-4919-9) discuss how fathers might influence the development of obesity and diabetes in their offspring.

Besides maternal nutrition, another in utero exposure that has been linked to offspring obesity and diabetes in later life is exposure to environmental toxicants. In their review, Sargis and Simmons (https://doi.org/10.1007/s00125-019-4940-z) discuss how endocrine-disrupting chemicals in the environment have been implicated in diabetes pathogenesis throughout the life course.

With regard to type 1 diabetes, Craig et al (https://doi.org/10. 1007/s00125-019-4942-x) look at how early life exposures contribute to type 1 diabetes risk. In terms of potential treatments for diabetes, Palmer et al (https://doi.org/10.1007/ s00125-019-4934-x) explore the biological links between ageing and diabetes, with a specific focus on cellular senescence. We conclude with a review by Timpel et al (https://doi.org/10.1007/s00125-019-4941-y) discussing what governments should be doing. The reviews are accompanied by an editorial (https://doi.org/10.1007/s00125-019-4954-6).

High maternal early-pregnancy blood glucose levels are associated with altered fetal growth and increased risk of adverse birth outcomes

Madelon L. Geurtsen, Eef E. L. van Soest, Ellis Voerman, Eric A. P. Steegers, Vincent W. V. Jaddoe, Romy Gaillard

Gestational diabetes mellitus (GDM) is a major risk factor for perinatal complications. Recent studies suggest that, in midand late pregnancy, high maternal glucose levels below the threshold of GDM are associated with increased risks of adverse birth outcomes. In this issue, Geurtsen et al (https://doi. org/10.1007/s00125-019-4957-3) performed a populationbased prospective cohort study of 6116 pregnant women to investigate the direct effects of impaired maternal glucose metabolism from early pregnancy onwards on fetal growth throughout pregnancy and on adverse birth outcomes. They 
report that higher maternal non-fasting glucose levels in early pregnancy are associated with altered fetal growth patterns, characterised by decreased fetal growth rates in midpregnancy and increased fetal growth rates from late pregnancy onwards, leading to an increased risk of delivering a largefor-gestational-age infant. The authors suggest that these findings indicate that instead of targeting maternal glucose metabolism in the second half of pregnancy, as is current clinical practice, future preventive strategies need to focus on screening for impaired maternal glucose metabolism from preconception and early pregnancy onwards. In addition, there is a need for intervention programmes to improve fetal growth and birth outcomes.

The effect of mid-life insulin resistance and type 2 diabetes on older-age cognitive state: the explanatory role of earlylife advantage

Sarah-Naomi James, Andrew Wong, Therese Tillin, Rebecca Hardy, Nishi Chaturvedi, Marcus Richards

Type 2 diabetes, hyperglycaemia and insulin resistance are considered risk factors for cognitive impairment. In this issue, James et al (https://doi.org/10.1007/s00125-019-4949-3) analysed data from the National Survey for Health and Development, a birth cohort established in 1946, to show that childhood advantage (childhood cognitive ability, socioeconomic status and education) drives this association, by separately benefitting older-age insulin resistance and cognition. They found no direct connection between the latter two variables. The authors used an alternative genetic approach, a polygenic risk score for insulin resistance, to confirm the lack of association with cognition. The authors conclude that these findings suggest that glucoselowering agents are unlikely to have a beneficial impact on cognitive decline and dementia, and that strategies to improve childhood circumstances may be better placed to impact both later life diabetes and cognitive impairment risk.

\section{The role of GIP and pancreatic GLP-1 in the glucoregulatory effect of DPP-4 inhibition in mice}

Chelsea R. Hutch, Karen Roelofs, April Haller, Joyce Sorrell, Kyle Leix, David D. D'Alessio, Robert Augustin, Randy J. Seeley, Thomas Klein, Darleen A. Sandoval

Several effective glucoregulatory pharmaceuticals target incretin peptides, such as glucagon-like peptide-1 (GLP-1) and glucose-dependent insulinotropic polypeptide (GIP). One such class of pharmaceuticals prolongs the half-life of these peptides by inhibiting the degradation enzyme, dipeptidyl peptidase-4 (DPP-4). It is commonly thought that the intestinal source of GLP-1 is responsible for improved glucose homeostasis by DPP-4 inhibitors. In this issue, Hutch et al (https://doi.org/10.1007/s00125-019-4963-5) studied transgenic mouse models in which GLP-1 is solely expressed in the pancreas or the intestine. Their findings indicate that pancreatic GLP-1 contributes to the effectiveness of DDP-4 inhibitors. In addition, in a mouse model with no GLP1 production, DPP-4 inhibitors retained a full glucoregulatory effect through the actions of GIP. The authors conclude that these findings suggest that increases in pancreatic GLP-1 and GIP are necessary for the full effect of DPP-4 inhibitors on glucose tolerance in mice.

Factors associated with stillbirth in women with diabetes

Sharon T. Mackin, Scott M. Nelson, Sarah H. Wild, Helen M. Colhoun, Rachael Wood, Robert S. Lindsay, on behalf of the SDRN Epidemiology Group and Scottish Diabetes Group Pregnancy subgroup

Stillbirth rates are up to fivefold higher in mothers with diabetes compared with those without. Identification of at-risk pregnancies is challenging for clinical teams. In this issue, Mackin et al (https://doi.org/10.1007/ s00125-019-4943-9) explored Scottish obstetric data to better identify the risk profile of affected pregnancies in women with type 1 and type 2 diabetes. In type 1, higher glycaemia throughout pregnancy was linked with stillbirth, whilst in type 2, higher pregestational glycaemia and maternal BMI had the strongest association, highlighting the importance of preconceptual preparation. Stillbirth risk was increased at the extremes of birthweight and, whilst the majority of stillbirths occurred preterm (under 37 weeks), one-third occurred at term. The authors conclude that more accurate prediction of pregnancies at risk of stillbirth is urgently needed.

Publisher's note Springer Nature remains neutral with regard to jurisdictional claims in published maps and institutional affiliations.

All text supplied by the authors. 